\title{
Auditory Nerve Fiber Discrimination and Representation of Naturally-Spoken Vowels in Noise
}

\author{
Amarins N. Heeringa and $\mathbb{C}^{C}$ Christine Köppl
}

https://doi.org/10.1523/ENEURO.0474-21.2021

Cluster of Excellence "Hearing4all" and Research Centre Neurosensory Science, Department of Neuroscience, School of Medicine and Health Science, Carl von Ossietzky University Oldenburg, Oldenburg 26129, Germany

\begin{abstract}
To understand how vowels are encoded by auditory nerve (AN) fibers, a number of representation schemes have been suggested that extract the vowel's formant frequencies from AN-fiber spiking patterns. The current study aims to apply and compare these schemes for AN-fiber responses to naturally-spoken vowels in a speech-shaped background noise. Responses to three vowels were evaluated; based on behavioral experiments in the same species, two of these were perceptually difficult to discriminate from each other (/e/ vs /i/), and one was perceptually easy to discriminate from the other two (/a:/). Single-unit AN fibers were recorded from ketamine/xylazine-anesthetized Mongolian gerbils of either sex $(n=8)$. First, single-unit discrimination between the three vowels was studied. Compared with the perceptually easy discriminations, the average spike timing-based discrimination values were significantly lower for the perceptually difficult vowel discrimination. This was not true for an average rate-based discrimination metric, the rate d-prime (d'). Consistently, spike timing-based representation schemes, plotting the temporal responses of all recorded units as a function of their best frequency (BF), i.e., dominant component schemes, average localized interval rate, and fluctuation profiles, revealed representation of the vowel's formant frequencies, whereas no such representation was apparent in the rate-based excitation pattern. Making use of perceptual discrimination data, this study reveals that discrimination difficulties of naturally-spoken vowels in speech-shaped noise originate peripherally and can be studied in the spike timing patterns of single AN fibers.
\end{abstract}

Key words: consonant-vowel-consonant logatomes; ICRA1 noise; Mongolian gerbil; OLLO speech material database; temporal coding

\section{Significance Statement}

Understanding speech in noisy environments is an everyday challenge. This study investigates how single auditory nerve (AN) fibers, recorded in the Mongolian gerbil, discriminate and represent naturally-spoken vowels in a noisy background approximating real-life situations. Neural discrimination metrics were compared with the known behavioral performance by the same species, comparing easy to difficult vowel discriminations. A spike-timing-based discrimination metric agreed well with perceptual performance, while mean discharge rate was a poor predictor. Furthermore, only spike-timing-based, but not the rate-based, representation schemes revealed peaks at the formant frequencies, which are paramount for perceptual vowel identification and discrimination. This study reveals that vowel discrimination difficulties in noise originate peripherally and can be studied in the spike-timing patterns of single AN fibers.

Received November 9, 2021; accepted December 17, 2021; First published January 27, 2022.

The authors declare no competing financial interests.
Author contributions: A.N.H. and C.K. designed research; A.N.H. performed research; A.N.H. analyzed data; A.N.H. and C.K. wrote the paper. 


\section{Introduction}

Vocal communication is indispensable for the survival and well-being of many species, including mammals. Speech is one of the more complex of such vocalizations that needs to be processed by the central auditory system. Nonetheless, humans, as well as behaviorally trained animals, are still well able to extract critical features of speech signals. Individuals can discriminate between different syllables or logatomes, short nonsensical pseudowords of one or two syllables, even when presented in challenging background sounds (Sinnott et al., 1997; Jürgens and Brand, 2009). This suggests that the mammalian auditory system is proficient at processing such temporally and spectrally complex signals.

Speech can be decomposed into vowels and consonants, in which vowels are spectrally, and consonants are temporally, more complex. In this study, we focused on the vowels. A vowel's spectrum comprises harmonics of the fundamental frequency (f0), which is the voice pitch, and two to five formant frequencies (f1-f5), which are global regions of maximum energy in the vowel spectrum (Diehl, 2008). The formant frequencies differ between different vowels, whereas the fo differs between speakers, but is similar between vowels spoken by the same speaker. For perceptual vowel discrimination, the formant frequencies are paramount; for example, whispered vowels, lacking fo, remain intelligible and discriminable. Hence for vowels to be discriminated, information on their formant frequencies needs to be encoded in the spike patterns of the auditory nerve (AN), the gateway between sensory hair cells of the inner-ear cochlea and the central auditory system.

To understand how the formant frequencies are encoded by the AN, previous research has suggested a number of analysis methods, or representation schemes, that can extract formant frequencies from $\mathrm{AN}$-spiking patterns. Vowel representation schemes for the AN include rate-based excitation patterns (Sachs and Young, 1979), dominant component schemes (Delgutte and Kiang, 1984a), averaged localized synchronized rate (ALSR) plots (Young and Sachs, 1979), and fluctuation profiles (Carney, 2018). Applying such representation schemes to the AN response to vowels presented in background noise, as well as to vowels presented at moderately high sound levels, results in degradation of neural formant representation, more in some schemes than in others (Young and Sachs, 1979; Sachs et al., 1983; Delgutte and Kiang, 1984b). Furthermore, in the search for such representation schemes, synthetic vowels were often used, in which

This work was supported by the Deutsche Forschungsgemeinschaft Cluster of Excellence 2177/1 "Hearing4all 2.0" Project ID 390895286.

Acknowledgements: We thank Rainer Beutelmann for his help with preparing the acoustic stimuli for use in our neurophysiology set up.

Correspondence should be addressed to Amarins N. Heeringa at amarins. nieske.heeringa@uni-oldenburg.de.

https://doi.org/10.1523/ENEURO.0474-21.2021

Copyright (C 2022 Heeringa and Köppl

This is an open-access article distributed under the terms of the Creative Commons Attribution 4.0 International license, which permits unrestricted use, distribution and reproduction in any medium provided that the original work is properly attributed. formant frequencies can coincide exactly with an fo harmonic and spectral changes over time do not occur. In synthesized vowels, a particular feature can be changed independently of others, to study its importance in vowel representation. For example, by applying a range of $\mathrm{f} 2$ frequencies, Conley and Keilson (1995) measured the minimal frequency difference that is represented in a ratebased excitation pattern. In addition, it has been shown that synthetic vowels presented in a consonant-vowel construct show improved rate representation in the transition between consonant to vowel (Sinex and Geisler, 1983). Naturally-spoken vowels, where the spectrum is rarely steady state over $>0.1 \mathrm{~s}$, however, pose an additional challenge to the representation schemes, especially when presented in background noise.

Ultimately, vowel representation in the AN only needs to be as good as the ability to perceptually discriminate between two highly similar vowels. Indeed, some vowels are perceptually easier to discriminate from each other than others, which is related to the degree of similarity in the formant frequencies (Jürgens and Brand, 2009). Therefore, knowledge of the discrimination performance between vowels provides crucial guidance about important vowel features and representation schemes, especially in challenging listening conditions.

Here, we probed the neural representation of naturallyspoken vowels from the Oldenburg Logatome (OLLO) database of speech material (Meyer et al., 2010) presented in a speech-shaped background noise, by applying previously developed representation schemes. Vowels were selected based on psychophysical behavioral experiments using the same species (gerbil) and the same speech material. In these experiments, animals were trained to perform an oddball target detection paradigm on vowels. A perceptual map was generated from the animal's behavioral response latencies using multidimensional scaling and shows difficult and easy discriminations as short and long distances, respectively. For the current study, we selected three vowels from this perceptual map. Thus, vowel discrimination could be compared between vowels that were easy and those that were difficult to perceptually distinguish by gerbils (Jüchter et al., 2021). Making use of perceptual discrimination data in challenging conditions, this study sheds light on the accuracy of different AN fiber representation schemes for revealing the encoding of naturally-spoken vowels in a speech-shaped background noise.

\section{Materials and Methods}

\section{Animals}

Single-unit AN recordings from eight young-adult (three to six months) Mongolian gerbils (Meriones unguiculatus) were used in this study. Animals weighed between 63 and $94 \mathrm{~g}$, were of either sex, and were free from middle-ear infections. Animals were born at the University of Oldenburg animal facility and were group housed (two to four litter mates of the same sex per cage) in a controlled, quiet environment. Experiments 
were conducted in a custom-build sound-attenuating chamber. Animals were anesthetized with an intraperitoneal injection containing a mixture of ketamine $(135 \mathrm{mg} / \mathrm{kg}$; Ketamidor, WDT) and xylazine (6 mg/kg; Xylazin, Serumwerk) diluted in saline $(0.9 \% \mathrm{NaCl})$. One-third of the initial ketamine/ xylazine dose was provided hourly or on a positive hind-paw reflex. Rectal body temperature was maintained at $38^{\circ} \mathrm{C}$ by a homeothermic blanket (Harvard Apparatus). Gerbils received oxygen flowing onto the snout ( $1.5 \mathrm{l} / \mathrm{min})$. The head of the animal was fixed in a bite bar (Kopf Instruments) and the right pinna was removed. The ear bar, containing a small speaker (IE 800, Sennheiser) and microphone (ER7-C, Etymotic Research), was sealed to the bony ear canal using petroleum jelly. A small opening in the bulla prevented the build-up of negative pressure in the middle ear cavity. To determine hearing thresholds before and during single-unit recordings, auditory brainstem responses (ABRs) to chirps $(0.3-19 \mathrm{kHz}, 4.2-$ ms duration, 300-500 repetitions) at a range of levels were recorded and visually evaluated. Experiments were terminated on an ABR threshold elevation of $>20 \mathrm{~dB}$, or on heart failure. All experimental procedures were performed in accordance with the animal ethics authorities of Lower Saxony, Germany (permit number AZ 33.19-42502-04-15/1990).

\section{Single-unit AN-fiber recordings}

The brainstem was exposed through a craniotomy of the right occipital bone, followed by a duratomy and partial aspiration of the cerebellum. The AN was visualized by placing small balls of paper tissue $(<0.5 \mathrm{~mm}$ in diameter), drenched in saline, between the brainstem and the temporal bone. Glass micropipette electrodes (GB120F-10, Science Products, pulled with a P-2000, Sutter Instruments) were filled with a $3 \mathrm{M} \mathrm{KCl}$ solution (21 to $44-\mathrm{M} \Omega$ impedance). Electrodes were advanced slowly through the AN bundle (6000 ULN inchworm motor controller and 6005 ULN handset, Burleigh), while playing a broadband-noise search stimulus (50$70 \mathrm{~dB}$ SPL) through the speaker, until a single unit was isolated. Neural signals were amplified (WPI 767, World Precision Instruments), filtered for line-frequency noise $(50 / 60 \mathrm{~Hz}$; Hum Bug, Quest Scientific), made audible through a speaker (MS2, TDT), visualized on an oscilloscope (SDS 1102CNL, SIGLENT Technologies), and digitized (RX6, TDT; $48828 \mathrm{~Hz}$ sampling rate) before storage on a personal computer using custom-written MATLAB software.

Tone bursts near $10 \mathrm{~dB}$ above the most sensitive threshold ( $50 \mathrm{~ms}$ ON-180 ms OFF time, 5 -ms cosine rise/ fall times, five repetitions, 10-20 linear frequency steps spanning around 1.5 octave) were presented to determine the unit's best frequency (BF). Subsequently, tone bursts at $\mathrm{BF}$ were presented at a range of levels $(3 \mathrm{~dB}$ step size, 10 repetitions) to determine the unit's threshold. When the unit was still stable after recording responses to the consonant-vowel-consonant (CVC) stimuli, $24 \mathrm{~s}$ of neural activity in the absence of acoustic stimuli were recorded to more accurately determine the unit's spontaneous rate (SR). When this recording was not available ( $40.5 \%$ of units), SR was estimated through the silent trials of the rate-level function recordings. All stimuli were calibrated using the
Table 1: Fundamental and formant frequencies and length of the analysis windows of the presented vowels

\begin{tabular}{lllll}
\hline & $\mathrm{f0}(\mathrm{Hz})$ & $\mathrm{f} 1(\mathrm{~Hz})$ & $\mathrm{f} 2(\mathrm{~Hz})$ & Analysis window $(\mathrm{ms})$ \\
\hline $\mathrm{F} / \mathrm{e} /$ & 256 & 400 & 2450 & 268 \\
$\mathrm{~F} / \mathrm{i} /$ & 260 & 300 & 2575 & 233 \\
$\mathrm{~F} / \mathrm{a} / /$ & 257 & 850 & 1275 & 269 \\
M /e/ & 107 & 300 & 1990 & 121 \\
M /i/ & 109 & 220 & 2040 & 110 \\
M /a:/ & 104 & 650 & 1150 & 143
\end{tabular}

Stimuli spoken by the female speaker are marked with $F$, stimuli spoken by the male speaker are marked with $\mathrm{M}$.

miniature microphone (ER7-C, Etymotic Research) sealed near the speaker in the ear bar, a microphone amplifier (MA3, TDT), and custom-made MATLAB software.

\section{CVC stimuli}

Vowels were presented in a CVC construct and derived from the OLLO speech-material database (Meyer et al., 2010). Figure $1 A$ shows an example of the first $0.7 \mathrm{~s}$ of the stimulus waveform /bahb/ in quiet (Fig. 1A, upper panel) and in $5 \mathrm{~dB}$ signal-to-noise ratio (SNR) noise (Fig. $1 A$, lower panel). The spectrogram of the same stimulus, in quiet, is shown in Figure $1 B$. For each CVC, an analysis window was defined, which included the steady-state section of the vowel (Fig. $1 A$, red square, $B$, white bar). CVCs were selected based on a behavioral study in gerbils from the same colony that had demonstrated common and uncommon confusions between all vowels available in the database (Jüchter et al., 2021). Three vowels were selected from that study: two that were difficult to discriminate from each other (/e/ vs /i/) and one that was easy to discriminate from the other two (/a:/; Fig. 1C). The outer consonant was fixed at $/ \mathrm{b} /$, resulting in the following spoken logatomes: /behb/, /bieb/, and /bahb/. CVCs were spoken by a single female speaker who was a native German speaker (noted S01F in the OLLO database). The fO, as well as the length of the vowel, which was used as an analysis window, differed slightly between the three vowels (see Table 1). In a control experiment, the same logatomes were used but spoken by a native German male speaker (S06M in the OLLO database). All CVCs were calibrated according to the recorded transfer function of the acoustic set up using custom-made MATLAB software. Formant frequencies ( $f 1$ and $f 2$ ) were derived from the vowel spectrum of the stimulus recorded with the microphone in the ear bar, i.e., the vowel spectrum of the stimulus delivered to the gerbil's ear (Table 1; Fig. 1D-F).

The total recording time of one presentation of a logatome was fixed at $1.15 \mathrm{~s}$. The logatome started after a delay of $125 \mathrm{~ms}$, had an average length of $570 \mathrm{~ms}$, and was presented at a fixed level of $65 \mathrm{~dB}$ SPL. Logatomes were presented against a speech-like background noise (ICRA1) derived from the ICRA database (Dreschler et al., 2001; Fig. $1 D-F$, gray traces). Calibrated noise was presented at a 5-dB SNR and mixed with the logatome before being presented through the same in-ear speaker. Each logatome was sequentially presented 60 times. 

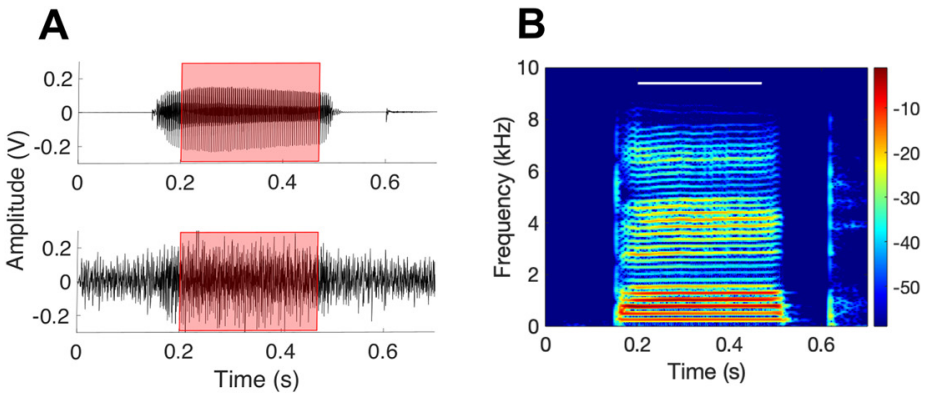

C
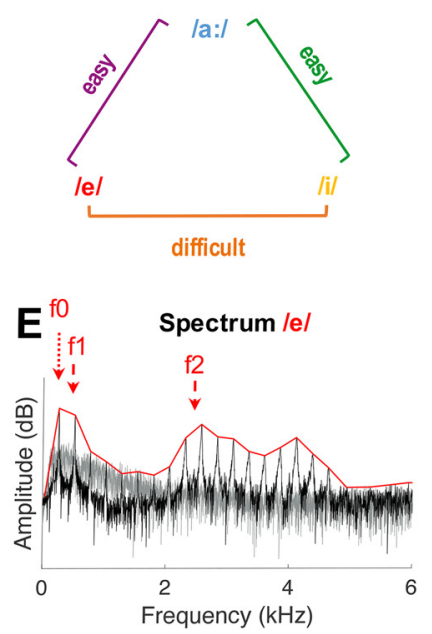

should be $>0.6 \mathrm{~ms}$ based on neural refractoriness (Heil et al., 2007). To confirm that the recorded unit was part of the AN bundle, and not of the cochlear nucleus, several additional checks were conducted. Spike waveforms were checked for the absence of a prepotential, the response pattern to tones at 20-30 dB above threshold were checked for having a primary-like shape, and the shape of the rate-level function was required to fall into one of the three known rate-level function shapes of the AN (straight, sloping saturating, or fast saturating; Winter et al., 1990). When responses to clicks were obtained, click latency was determined and matched to our own click latency versus BF distribution of AN fibers. When any of these checks failed, the unit was excluded from further analysis.

\section{Vowel discrimination}

Responses to vowels were included in the analysis when a minimum of 20 successful trials were recorded and a minimum of 200 spikes were evoked during vowel presentation. Responses of vowels in noise were pooled across the two different frozen background noises, but not across different speakers. For the female speaker, a total of 39 units were included in the study and in $n=23$ of these units, responses to all vowels could be recorded and included in analyses. For the male speaker, a total of 15 units were included and in $n=12$ units responses to all vowels were obtained. There were no units in which responses to both the female and the male speaker were recorded. Table 2 gives an overview of the number of units for each vowel and vowel combination.

In total, three comparisons were made, two behaviorally easy comparisons (/a:/ vs /e/ and /a:/ vs /i/) and one behaviorally difficult comparison (/e/ vs /i/). Rate-based vowel discrimination by single-unit AN fibers was determined by calculating d-prime (d') over the mean $(\mu)$ and SD $(\sigma)$ of the firing rates in response to two vowels, according

Noise was frozen between repetitions, as well as between different units. A total of two different frozen noises were presented for one complete set of stimuli. Noise onset and offset were ramped with a 10-ms cosine squared window at the start and end of the recording time.

\section{Data analysis}

Neural signals were bandpass filtered between 0.3 and $3 \mathrm{kHz}$. The threshold for spike detection was revisited offline for all recordings on a trial-by-trial basis, as spike amplitude could vary during recordings. Singleunit isolation was confirmed by checking interspike intervals, of which a large majority should be $>1 \mathrm{~ms}$ and all

to the following equation:

$$
d^{\prime}=\frac{\mu_{1}-\mu_{2}}{\sqrt{\left(\sigma_{1}^{2}+\sigma_{2}^{2}\right) / 2}} .
$$

$A\left|d^{\prime}\right|=1$ means that the mean firing rates in response to the two vowels are $1 \mathrm{SD}$ apart, which is typically considered to be a just-noticeable difference between two responses and can be used as a discrimination threshold (Green and Swets, 1974; Conley and Keilson, 1995).

Spike timing-based discrimination of vowels was based on the shuffled and crossed autocorrelograms of responses to one vowel and between two vowels, respectively (Joris, 
Table 2: Numbers of units from which responses to vowels were recorded

\begin{tabular}{llll}
\hline Single vowels & /a:/ & /e/ & /i/ \\
\hline Number of units female speaker & 39 & 33 & 24 \\
Number of units male speaker & 13 & 15 & 15 \\
& & & \\
Vowel discriminations & /a:/ vs /e/ & /a:/ vs /i/ & /e/ vs /i/ \\
Number of units female speaker & 33 & 24 & 23 \\
Number of units male speaker & 12 & 12 & 14
\end{tabular}

Unit numbers are given for the responses to a single vowel (data in Figs. 6, 7, $9,10)$ and for responses to a combination of two vowels (data in Figs. 2-4). Unit numbers for responses to vowels spoken by the female and male speaker are presented separately.

2003; Louage et al., 2004). Autocorrelograms were constructed using a bin width of $20.48 \mu$ s and were normalized for firing rate, repetition number, bin width, and analysis window. The shortest analysis window length (for /i/, see Table 1) was applied to all vowels in this analysis. The maximum peak height of the shuffled and crossed autocorrelogram is an indication of trial-by-trial temporal similarity and is defined as the correlation index $(\mathrm{Cl})$ and the cross- $\mathrm{Cl}(\mathrm{Clx})$, respectively. Hence, the $\mathrm{Clx}$ indicates the similarity of the temporal responses to the two vowels that are discriminated, with low values meaning low similarity and thus suggesting good discrimination. However, if the $\mathrm{Cl}$, i.e., the temporal similarity of the response to one vowel, is already low, the Clx is also expected to be low which, in this case, is meaningless re. discriminability. Therefore, the difference between $\mathrm{Cl}$ and $\mathrm{Clx}$ will be taken as a temporal discrimination metric, as follows:

$$
\Delta C l=C l-C l x .
$$

A high $\Delta \mathrm{Cl}$ value reflects temporal dissimilarity between responses to the two vowels in the comparison, whereas a low $\Delta \mathrm{Cl}$ value reflects temporally similar responses to the two vowels, regardless of the overall temporal precision of spiking of a given AN fiber.

\section{Dominant component scheme}

An all-order interspike interval histogram (ISIH) was constructed based on spike times during presentation of the vowel (Table 1). Only intervals shorter than 31.25 ms were included in the histogram, which consisted of 256 bins, resulting in a bin width of $0.12 \mathrm{~ms}$. The histogram was converted to interval rate by multiplying the probability of obtaining an interval that falls into a certain bin with the average discharge rate (Sachs and Young, 1980). A fast-Fourier transform (FFT) of the all-order ISIH revealed responses to the different frequencies. The dominant component scheme was constructed by plotting the frequency of the highest peak in the FFT of the ISIH against the unit's BF.

\section{Averaged localized interval rate (ALIR)}

To compute the ALIR, the average peak height of the ISIH FFT at each harmonic of f0 was calculated for all units tuned at or close to that harmonic (Sachs and Young, 1980). A range of $+/-0.5$ octave around each harmonic was taken to determine for which units the ALIR was computed. This results in the following equation to compute the ALIR:

$$
A L I R(k)=\frac{1}{N_{k}} \sum_{l=1}^{N_{k}} H_{k l},
$$

in which $N_{k}$ is the number of fibers tuned within $+/-0.5$ octaves of the $k^{\text {th }}$ harmonic, $I$ is the fiber number within that set of fibers, and $H_{k l}$ is the ISIH FFT peak height of the $l^{\text {th }}$ fiber at the $k^{\text {th }}$ harmonic of fo. Subsequently, the ALIR of each vowel response was plotted as a function of frequency of the fo harmonics.

\section{Fluctuation amplitude profile}

Fluctuation amplitude profiles were constructed using the peristimulus time histogram (PSTH) of the neural response to the vowel (Carney, 2018). To capture f0 fluctuations in the PSTH, bin width was defined as eight bins per f0 period (1/8 $\times \mathrm{fO})$. The amplitude of the fluctuation was determined using the coefficient of variation (CV), CV $=\sigma_{n} / \mu_{n}$, in which $n$ refers to the bin height of the PSTH in spikes per second. Subsequently, the fluctuation amplitude was also established by calculating the rate change of the PSTH, i.e., the mean absolute difference between each bin height divided by the mean bin height. CV and rate change values close to 0 indicate little to no fluctuation, i.e., putative formant frequency encoding. The fluctuation amplitude was plotted as a function of the fiber's BF to obtain the fluctuation amplitude profile.

\section{Rate-based excitation pattern}

The rate-based excitation pattern plots the vowel-evoked normalized rate as a function of the fiber's BF (Sachs and Young, 1979). The average firing rate during the vowel was normalized for the fiber's SR and saturation rate as follows:

$$
\text { Normalized rate }=\frac{\text { rate }-S R}{\text { saturation rate }-S R} .
$$

The saturation rate was derived from the rate-level function as the firing rate at $50 \mathrm{~dB}$ above threshold, according to Sachs and Young (1979).

\section{Statistics}

Rate-based and spike-timing-based discrimination was compared between the different vowel combinations using a one-way repeated-measures ANOVA (RM-ANOVA), followed by post hoc paired $t$ tests. The Bonferroni-Holm correction was applied to multiple comparisons. Frequency distributions of the dominant components derived from the ISIH FFTs were compared between responses to the different vowels using a Kolmogorov-Smirnov test. Statistical analyses were conducted in MATLAB using the Statistics and Machine Learning Toolbox. Data were presented separately for low-SR and high-SR fibers, with the cutoff between these populations at 18 spikes/s (Schmiedt, 1989). 

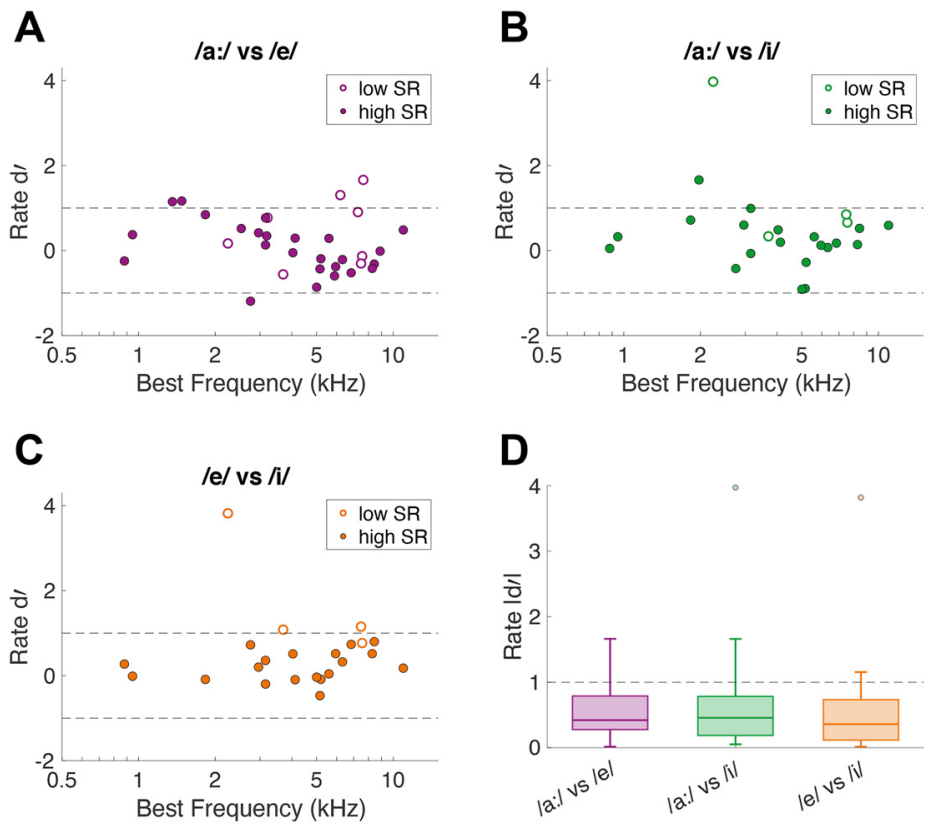

Figure 2. Single-unit AN-fiber vowel discrimination based on average firing rate. $\boldsymbol{A}-\boldsymbol{C}, \mathrm{d}$ ' values for each unit's firing rate in response to the two vowels that are compared, plotted as a function of the unit's BF. Low-SR and high-SR fibers are plotted with open and closed symbols, respectively. Dashed horizontal lines indicate a neural discrimination threshold of $\left|d^{\prime}\right|=1$. Number of units (of those, number of low-SR units) included in the vowel comparisons are $n=33$ (8), $n=24$ (4), and $n=23$ (4) for panels $\boldsymbol{A}, \boldsymbol{B}$, and $\boldsymbol{C}$, respectively. $\boldsymbol{D}$, Absolute d'values of all units presented in boxplots. The dashed horizontal line indicates $\left|d^{\prime}\right|=1$.

\section{Results}

\section{Perceptual and neural discrimination of vowels agree when using a spike timing-based but not when using a rate-based discrimination metric}

Discrimination between vowels in neural responses of single AN fibers was studied using rate-based and spike timingbased discrimination metrics. To determine whether mean firing rate differed significantly between responses to different vowels, d' based on firing rate during the vowel presentation was calculated. Most d' values were between -1 and 1 for all three comparisons (/a:/ vs /e/, /a:/ vs /i/, and /e/ vs /i). This indicates that for most units, the firing rate did not differ significantly between responses to the different vowels (Fig. 2A-C). Furthermore, low-SR and high-SR units both showed a similar (small) fraction of $\left|d^{\prime}\right|$ values $>1$ for all three comparisons (Fig. $2 A-C$ ). The BF of units with $\left|d^{\prime}\right|>1$ did not correspond to formant frequencies of either vowel in the comparison. Importantly, absolute d', averaged over all units, did not significantly differ between the perceptually easy (/a:/ vs /e/ and /a:/ vs /i/) and the difficult (/e/ vs /i/) comparisons (RMANOVA: $F_{(2,44)}=0.76, p=0.47$; Fig. $\left.2 D\right)$, indicating that the rate d' of neural responses cannot predict perceptual vowel discrimination.

The spike timing-based neural discrimination metric was calculated using auto- and cross-correlation indices. For the resulting $\Delta \mathrm{Cl}$, low-SR fibers achieved much higher maximal values compared with high-SR fibers, which was evident for all three comparisons (Fig. $3 A-C$ ). Furthermore,
$\Delta \mathrm{Cl}$ values differed across $\mathrm{BF}$, such that units with a $\mathrm{BF}<3 \mathrm{kHz}$ and $>8 \mathrm{kHz}$ had higher $\Delta \mathrm{Cl}$ values. Most importantly, average $\Delta \mathrm{Cl}$ differed significantly between the vowel comparisons (RM-ANOVA: $F_{(2,90)}=16.07, p=1.07 \times 10^{-6}$; Fig. 3D). Specifically, the difficult comparison (/e/ vs /i/) revealed significantly lower $\Delta \mathrm{Cl}$ values compared with both perceptually easy comparisons (/a:/ vs /e/, post hoc paired $t$ tests: $t_{(35)}=3.51, \quad p=0.0021 ; \quad / a: /$ vs $/ \mathrm{i} /$, $t_{(45)}=4.98, p=2.93 \times 10^{-5}$, Bonferroni-Holm corrected).

To examine whether this result was influenced by specific features of the background noise, we repeated the same analysis, comparing $\Delta \mathrm{Cl}$ when vowels were presented against two different background noises. Compared with the two easy comparisons, $\Delta \mathrm{Cl}$ was again lower in both conditions for the perceptually difficult comparison (/e/ vs /i). This difference was significant for noise \#1 (RM-ANOVA: $F_{(2,50)}=11.45, p=8.03 \times 10^{-5}$; Fig. $4 A$ ) and for noise \#2 (RM-ANOVA: $F_{(2,38)}=4.82, p=0.014$; Fig. $\left.4 B\right)$. We were also interested to observe whether vowels spoken by a different speaker, with a different fO, would yield the same outcome. Therefore, we collected responses from 17 additional units to CVCs spoken by a male speaker, all presented against background noise \#1. For the male speaker, too, the perceptually difficult comparison showed lower $\Delta \mathrm{Cl}$ values compared with the easy comparisons (RM-ANOVA: $F_{(2,46)}=11.71, p=7.75 \times$ $10^{-5}$; Fig. $4 C$ ). Additionally, the two easy comparisons were also significantly different from each other in noise \#2 and for the male speaker.

These results imply that spike-timing information in ANfiber spiking patterns is important to discriminate between naturally-spoken vowels in background noise. The same is intuitively apparent by contrasting AN rate and timing responses to the naturally-spoken vowels in quiet and in the speech-shaped background noise (Fig. 5), a comparison that drastically highlights the added difficulty of discrimination in the noisy condition and the concurrent importance of spike timing. The results presented in this paragraph together suggest that formant representation of these vowels by the AN-fiber population is likely to be based on spike timing. In the following paragraphs, we will test this hypothesis.

\section{Spike timing-based representation schemes can differentiate formant frequencies of naturally-spoken vowels in noise}

The dominant component scheme, first proposed by Delgutte and Kiang (1984a), is an overview of the dominant frequency component in the temporal spiking response to the vowel by a population of AN fibers across different BF. The dominant component is typically derived from a Fourier transform of the f0 phase histogram (PH; Fig. 6A). However, with naturally-spoken vowels, f0 
A

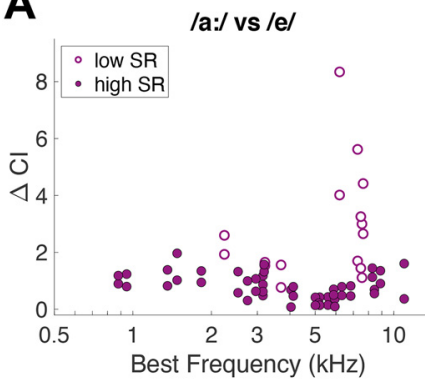

C

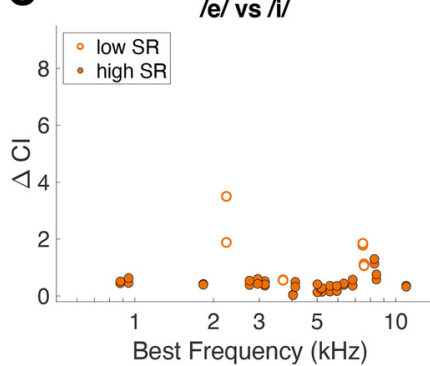

B

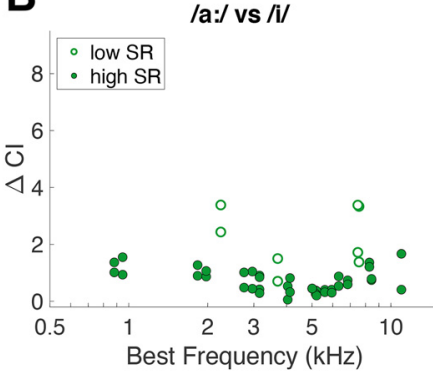

D

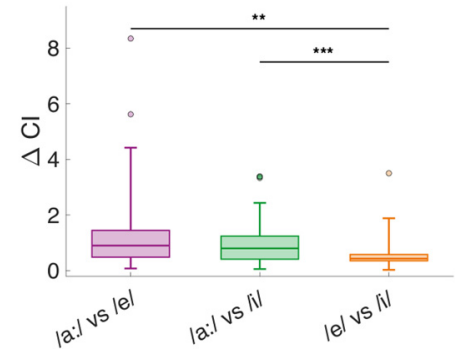

Figure 3. Single-unit AN-fiber vowel discrimination based on spike timing. $\boldsymbol{A}-\boldsymbol{C}$, $\Delta \mathrm{Cl}$ values for each unit in response to the two vowels that are compared, plotted as a function of the unit's BF. Low-SR and high-SR fibers are plotted with open and closed symbols, respectively. Number of units (of those, number of lowSR units) included in the vowel comparisons are $n=33$ (8), $n=24$ (4), and $n=23$ (4) for panels $\boldsymbol{A}, \boldsymbol{B}, \boldsymbol{C}$, respectively. Note that there are two values per unit in this analysis. $\boldsymbol{D}, \Delta \mathrm{Cl}$ of all units presented in boxplots. Asterisks indicate a significant difference, based on post hoc paired $t$ tests $\left({ }^{* *} p<0.01,{ }^{* \star *} p<0.001\right)$.

may change over time. Furthermore, formant frequencies can be located between two f0 harmonics, instead of precisely coinciding with an fo harmonic. To account for this natural variability, the frequency of the largest peak in the Fourier transform of the all-order ISIH was used to construct the dominant component scheme (Fig. 6B).

Figure $6 C-E$ shows the dominant component scheme, based on the all-order ISIH Fourier transform, of the ANfiber responses to the three naturally-spoken vowels in noise. Formant representation in the dominant component schemes differed between the different vowels. Responses to the vowel /a:/ were all dominated by temporal responses to the third and fourth fo harmonics, as such likely representing $\mathrm{f} 1$ and $\mathrm{f} 2$. Harmonic responses to $\mathrm{f} 1$ and $\mathrm{f} 2$ were represented by a subset of fibers for the responses to /e/ and /i/, leaving some fibers also responding to f0 or to frequencies between formants and fO harmonics. There were no apparent differences between low-SR and high-SR units in the dominant component schemes. When comparing the frequency distribution of the dominant components, all three distributions were significantly different from each (KolmogorovSmirnov tests: /a:/ vs /e/, $D=0.85, p=6.53 \times 10^{-12}$; /a:/ vs li $/, D=0.92, p=8.07 \times 10^{-12} ; / \mathrm{e} /$ vs $/ \mathrm{i} /, D=0.64, p=8.93 \times$ $10^{-6}$; Fig. $6 F$ ). This agrees with the fact that all three vowels can be perceptually distinguished from each other (Jüchter et al., 2021). Nevertheless, out of all three comparisons, the KS statistics and p-value was smallest for the /e/ vs /i/ comparison, following expectations based on perceptual discrimination measures.

Since one component may be dominant in many fibers, it has been proposed taking the average peak height of the fO PH FFT or of the ISIH FFT only of fibers that are tuned at or close to the respective f0 harmonic (Sachs and Young, 1980; Voigt et al., 1982). The resulting ALSR or ALIR, respectively, is a measure derived from the peak amplitudes of the FFTs (Fig. 6A,B). At specified frequency steps around each harmonic of $\mathrm{fO}$, the average of this amplitude is taken for all units tuned to this fo harmonic $+/-0.5$ octaves. Plotting the ALSR or ALIR as a function of the respective fo harmonic reveals a spectrum that has been shown to be robust against background noise, stimulus level, and inharmonic, whispered vowels (Young and Sachs, 1979; Voigt et al., 1982; Sachs et al., 1983). Figure 7 shows the ALIRs derived from combined neural responses of low-SR and high-SR fibers to the three naturally-spoken vowels in noise. The current dataset did not cover BFs at all harmonics of f0, explaining the missing points in the ALIR. Responses to vowel /a:/ showed a dominant peak at the fourth f0 harmonic, representing formant frequencies $\mathrm{f} 1$ and $\mathrm{f} 2$ (Fig. 7A). The ALIR of responses to /e/ showed a trough at frequencies between $\mathrm{f} 1$ and $\mathrm{f} 2$, and $a$ clear peak at the 10th f0 harmonic, representing f2 (Fig. 7B). Likely because of a lower number of data points (see Table 2), the ALIR of responses to the vowel /i/ does not represent the formant frequencies clearly. Low-SR fibers contributed only marginally to these schemes, since most low-SR fibers had a $\mathrm{BF}>4 \mathrm{kHz}$, which is the upper frequency border of these ALIRs and also above of the phase-locking range for gerbil AN fibers (Versteegh et al., 2011).

A more recently developed representation scheme of neural vowel encoding is the fluctuation amplitude profile (Carney, 2018; Scheidiger et al., 2018). This scheme proposes that slow rate fluctuations to frequencies around f0 are strongest for fibers with a BF below, between, or above the formant frequencies, whereas fibers tuned at the formants display a more constant rate response, i.e., a less fluctuating PSTH. The degree of fluctuation was derived from the PSTH of the vowel response. Figure 8 shows an example of a fiber with high and a fiber with low fluctuation amplitudes to the vowel /e/ (Fig. 8A,B, respectively). Fluctuation amplitude was expressed as RC (rate change) and CV. Indeed, the response with visually high fluctuation amplitudes had a RC and $C V$ value that was about twice as high compared with the response with visually low fluctuation amplitudes. The high fluctuation amplitude response was recorded from a fiber tuned far from the formant frequencies $(B F=8.3 \mathrm{kHz}$; Fig. $8 A$ ), whereas the low fluctuation response was recorded from a fiber 


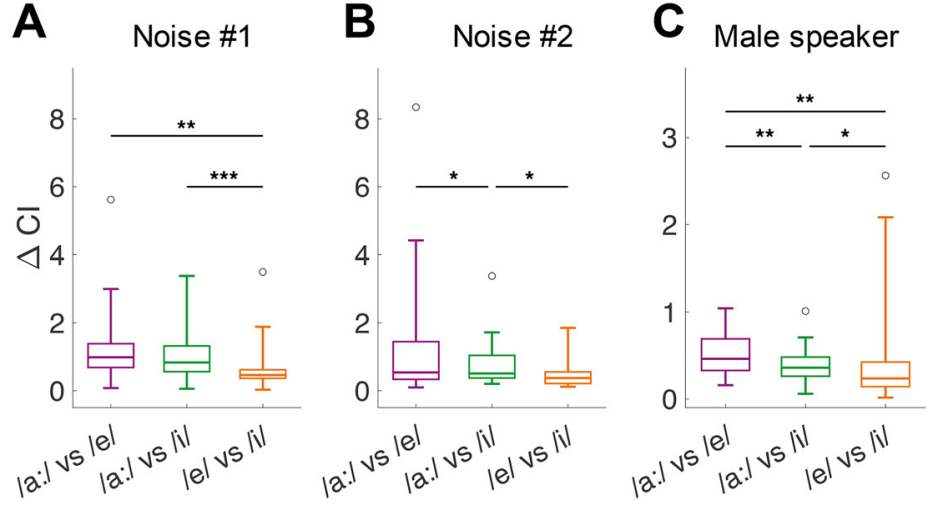

Figure 4. Effects of background noise and speaker-specific characteristics on spike-timing-based vowel discrimination. $\boldsymbol{A}, \Delta \mathrm{Cl}$ values for vowel comparisons presented against background noise \#1 at $5 \mathrm{~dB}$ SNR, spoken by a female speaker of the OLLO database (S01F). Number of units are $n=20$ for /a:/ vs /e/, $n=13$ for /a:/ vs /i/, and $n=13$ for /e/ vs /i/. B, $\Delta$ Cl values for vowel comparisons presented against noise \#2 (5 dB SNR, S01F speaker of the OLLO database). Number of units are $n=13$ for /a:/ vs /e/, $n=11$ for /a:/ vs /i/, and $n=10$ for /e/ vs /i/. C, $\Delta \mathrm{Cl}$ values for vowel comparisons spoken by a male speaker of the OLLO database (S06M), presented against noise \#1. Number of units are $n=12$ for /a:/ vs /e/, $n=12$ for /a:/ vs /i/, and $n=14$ for /e/ vs /i/. Asterisks indicate a significant difference, based on post hoc paired $t$ tests after the RM-ANOVA revealed a significant difference between the comparisons $\left({ }^{\star} p<0.05\right.$, ${ }^{* \star} p<0.01$, $\left.{ }^{\star \star *} p<0.001\right)$.

tuned near $\mathrm{f} 2$ of the vowel /e/ (BF $=2.8 \mathrm{kHz}$; Fig. $8 B)$. Both fibers had a high SR, to ensure a valid comparison, as low-SR fibers typically showed higher fluctuation amplitudes in general (see below). Fluctuation profiles were obtained by plotting the CV (Fig. $9 A-C$ ) and the rate change of the fiber's vowel response (Fig. $9 D-F$ ) as a function of the fiber's BF. Since less fluctuation hypothetically corresponds to better formant representation, the ordinate is plotted in reverse direction. Both methods showed similar patterns. Low-SR and high-SR fiber responses suggested two separate populations, with lowSR fibers showing higher CVs and rate change compared with the general trend of the high-SR fibers. For responses to the vowel /a:/, there was no clear formant-related peak in the fluctuation profile of the high-SR fibers, indicated by the moving average over the high-SR fibers in the CV and rate change (solid lines in Fig. $9 A, D$, respectively). The low-SR fibers appeared to contribute more to the fluctuation profile, by showing large fluctuations at high frequencies, but the number of low-SR fibers was too small to draw conclusions. For responses to the vowels /e/ and /i/, the moving averages of the high-SR fibers suggested a peak at $\mathrm{f} 2$ in the fluctuation amplitude profiles (Fig. 9B,C,E,F). No fibers with a BF at or around $f 1$ of $/ \mathrm{e} /$ and $/ \mathrm{i} /$ were recorded. In the fluctuation profiles of /e/ and $/ \mathrm{i} /$, low-SR fibers similarly seemed to enhance the fluctuation profile of the high-SR fibers, but are too scarce to make conclusive statements.

\section{The rate-based excitation pattern does not represent formant frequencies of naturally-spoken vowels in noise}

To cross-check for representation of the three vowels in the average firing rate response, we also plotted the normalized rate-based excitation pattern (Sachs and Young, 1979). Vowel-evoked firing rates, normalized for each fiber's spontaneous and saturation rate, was highly variable across the fibers and did not represent formant frequencies of the naturally-spoken vowels in noise (Fig. $10 A-C)$. Furthermore, there were no apparent differences in normalized rate between high-SR and low-SR fibers for any of the vowel responses.

\section{Discussion}

The current study showed that discrimination between vowel-evoked responses of single AN fibers agreed with perceptual discrimination abilities when analyzed using a spike timing-based discrimination metric. In contrast, no differences between easy and difficult comparisons were observed when considering a rate-based discrimination metric. Consistently, spike timing-based representation schemes of the AN population response revealed representation of the vowel's formant frequencies, whereas no such representation was apparent in a rate-based excitation pattern. Formant frequencies were differentially represented for the different vowels in the representation schemes and low-SR fibers comprised a separately responding group of fibers in their temporal responses. The findings were based on responses to vowels presented in challenging listening conditions, i.e., naturally spoken and presented in $5 \mathrm{~dB}$ SNR speech-shaped background noise.

\section{Temporal spiking information in AN fibers is suited to encode naturally-spoken vowels in background noise}

Early research on the neural encoding of vowels showed that a rate-based excitation pattern, using the normalized rate as in the current study, reveals peaks at fibers tuned to formant frequencies (Sachs and Young, 1979). However, rate-based excitation patterns are not robust against a few critical situations. When vowels are presented at levels higher than 40-65 dB SPL, depending on the vowel, formant peaks disappear (Sachs and Young, 1979). Furthermore, formant peaks in the rate-based excitation patterns also disappear when vowels are presented in background noise (Sachs et al., 1983; Delgutte and Kiang, 1984b). The effects of moderate-to-high sound levels and of background noise on the rate response to vowels can be explained by two nonlinearities in the rate responses of AN fibers, namely rate saturation and two-tone suppression (Sachs and Young, 1979, 1980). Especially two-tone suppression, i. e., suppression of the firing rate to tones at BF by lower-frequency tones, was suggested to be responsible for the flattening of the rate-based excitation pattern when vowels were presented at high sound levels and in noise (Sachs et al., 1983). Our data are consistent with these findings, showing no apparent formant- 

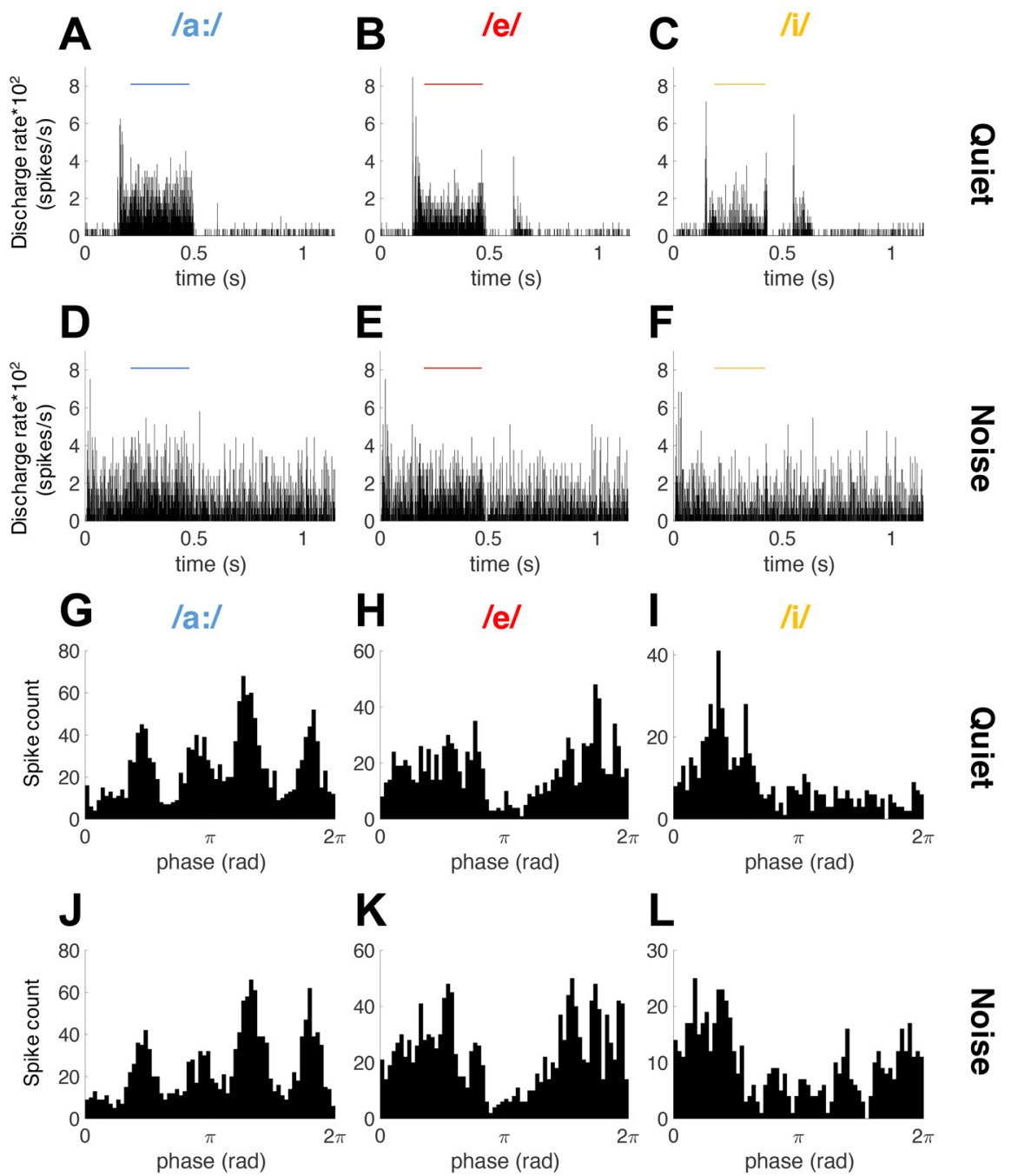

Figure 5. Example of one fiber responding to the three logatomes in quiet and in noise. PSTH of the response to the complete stimulus in quiet $(\boldsymbol{A}-\boldsymbol{C})$ and in $5 \mathrm{~dB}$ SNR speech-shaped ICRA1 noise $(\boldsymbol{D}-\boldsymbol{F})$. The vowel analysis window is indicated with a horizontal bar. Bin width is $0.49 \mathrm{~ms}$ for the graphs in panels $\boldsymbol{A}-\boldsymbol{F}$. PH of F0 during the vowel analysis window in quiet (G-I) and in $5 \mathrm{~dB}$ SNR noise $(\boldsymbol{J}-\mathbf{L})$. PHs were plotted with 64 bins per f0 cycle. This fiber had a BF of $2.2 \mathrm{kHz}$, a threshold at $28 \mathrm{~dB}$ SPL, and a SR of 4.7 spikes/s. Note the remarkably little effect of noise on the shapes of the PHs after the addition of background noise (panels $J-L$ ).

related peaks in the normalized rate-based excitation pattern of vowels presented in background noise (Fig. 10). Single-fiber discrimination abilities based on rate revealed significant changes in a few fibers for all three vowel comparisons. However, these high d'values did not appear to relate to the formant frequencies, in contrast to that shown for formant frequency shifts in synthetic vowels presented in quiet (Conley and Keilson, 1995). Importantly, average d' values over all units did not distinguish between perceptually difficult and perceptually easy vowel discriminations (Fig. 2D), based on a behavioral study using the same stimuli and the same animal species (Jüchter et al., 2021). This suggests that the average firing rate of AN fibers is not crucial for encoding naturally-spoken vowels in background noise.

In contrast, discrimination of vowel responses by a spike timing-based metric did agree with expectations from behavioral studies (Fig. 3D). Specifically, the perceptually difficult discrimination (/e/ vs /i/) also evoked the temporally most-similar spiking patterns in AN fibers, compared with the perceptually easy discriminations (/a:/ vs /e/ and /a:/ vs /i/). In agreement with the temporally based discrimination at the single-unit level, representation schemes that were based on spike-timing patterns, i.e., the dominant component scheme, the ALIR, and the fluctuation profile, represented peaks at most formant frequencies of the naturally-spoken vowels in noise.

It has been shown previously that the dominant component scheme, as well as the ALIR, is robust against higher sound levels (Young and Sachs, 1979; Delgutte and Kiang, 1984a), as well as against background noise (Sachs et al., 1983; Delgutte and Kiang, 1984b). Both these representation schemes are based on phaselocking strength, on the frequencies that are encoded by the temporal spiking patterns, and on the BF of the fiber. Since the vowels for this study were naturally 
A

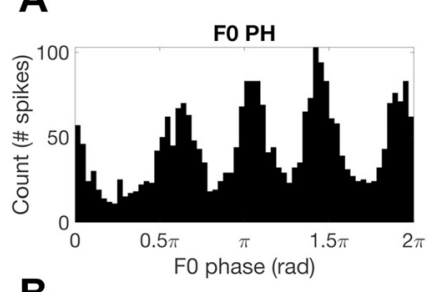

B
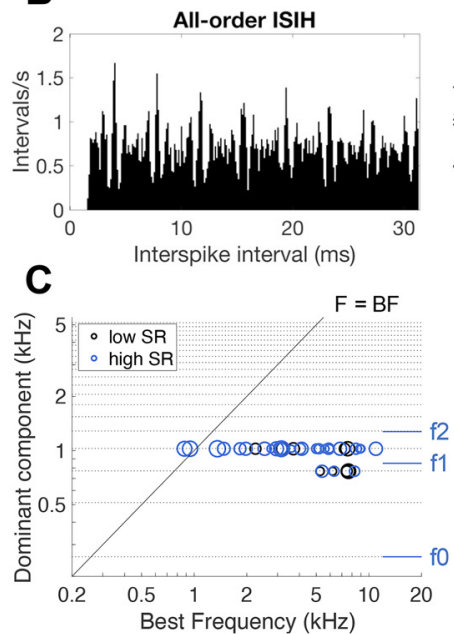

E

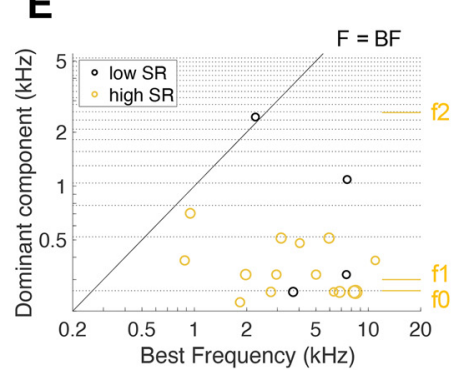

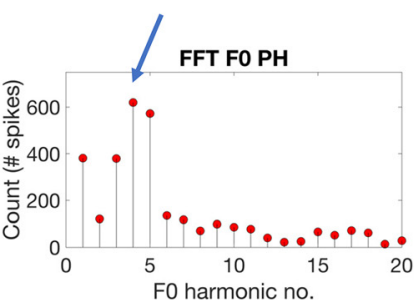
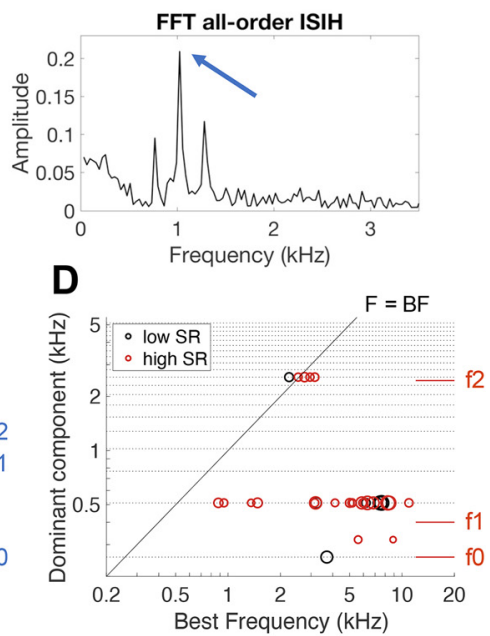

$\mathbf{F}$

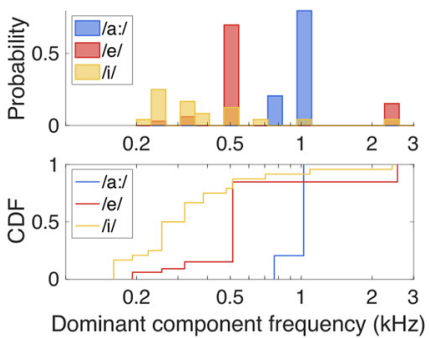

Figure 6. Dominant component schemes. A, Response of an AN fiber to the vowel /a:/ plotted in a $\mathrm{PH}$ for one period of fO. The FFT of the fO $\mathrm{PH}$ is plotted next to it. The arrow indicates the highest peak in the FFT of the fO PH at the fourth harmonic of $\mathrm{fO}$ and its relative peak height plotted in number of spikes. This example fiber was a high-SR fiber (46 spikes/s) with a BF at $1.3 \mathrm{kHz}$. B, All-order ISIH of the same data presented in panel $\boldsymbol{A}$, accompanied by its FFT. The arrow indicates the highest peak in the FFT, at $1024 \mathrm{~Hz}$, which corresponds to the frequency of the fourth harmonic of fo. $\boldsymbol{C}-\boldsymbol{E}$, Dominant component schemes plotting the frequency of the highest peak in the FFT of the all-order ISIH (panel $\boldsymbol{B}$ ) as a function of the fiber's BF for responses to the vowels /a:/ [panel C, $n=39$ ( $n=10$ low-SR fibers)], /e/ [panel $\boldsymbol{D}$, $n=33$ ( $n=8$ low-SR fibers)], and /i/ [panel $\boldsymbol{E}, n=24$ ( $n=4$ low-SR fibers)]. Formant frequencies are indicated on the right and the $\mathrm{F}=\mathrm{BF}$ line is indicated as a solid black line. Horizontal dotted lines indicate frequencies of fo harmonics. Symbol size reflects the height of the peak in the ISIH FFT, which is a measure of the strength of the temporal coding at the respective frequency component. $\boldsymbol{F}$, Frequency distributions of dominant components for responses to /a:/, /e/, and /i/ in blue, red, and yellow, respectively. Data are plotted in a histogram (upper panel) as well as in the form of a cumulative distribution function (CDF; lower panel).

spoken, the classical representation, based on the f0 $\mathrm{PH}$ and its Fourier transform (Sachs and Young, 1979; Delgutte and Kiang, 1984a), was less sensitive for the higher fo harmonics. Therefore, we used the ISIH to determine phase-locking frequencies of the single-fiber responses (Sachs and Young, 1980). This method has previously proven useful for voiceless, whispered

vowels, which lack f0 and its harmonics (Voigt et al., 1982). By constructing an all-order ISIH, compared with a first-order ISIH as in most studies, the resolution of the FFT and its relevant peaks became sufficiently high. Experimental data showing fluctuation profiles of AN fiber responses to vowels have not been reported elsewhere. Models predict less fluctuation in the PSTH for fibers tuned at or close to the formant frequencies and more fluctuation for fibers tuned to frequencies inbetween or above the formant frequencies (Carney, 2018; Scheidiger et al., 2018). A broad peak at $\mathrm{f} 2$ was observed in the fluctuation profiles of responses to vowels /e/ and /i/. Therefore, we showed for the first time that fluctuation profiles can be used to reveal some formant frequencies of spoken vowels in background noise from AN responses. Rate change appeared to be a slightly stronger metric compared with the $\mathrm{CV}$, but both revealed the same patterns for all vowel responses.

All spike timing-based representation schemes applied in this study revealed formant frequencies for one or more vowels. Hence, it was unimportant precisely which temporal metric was chosen, as the principal conclusion generalizes.

\section{Low-SR and high-SR fibers follow separate distributions for temporally- based encoding of vowels}

SRs of AN fibers vary widely between fibers, ranging from 0 to 150 spikes/s, and have been shown to correlate to physiological and morphologic properties of the fibers (Liberman, 1978, 1982; Schmiedt, 1989; Merchan-Perez and Liberman, 1996; Huet et al., 2016). In terms of rate encoding, high-SR fibers typically saturate over a narrower range of sound levels (around $20 \mathrm{~dB}$ ) compared with low-SR fibers (Winter et al., 1990). Furthermore, low-SR fibers generally have higher thresholds than high-SR fibers (Liberman, 1978; Huet et al., 2016). These characteristics are likely to be responsible for the fact that rate-based excitation patterns of low-SR (but not high-SR) fibers are more robust, even at higher sound levels (Conley and Keilson, 1995). However, low-SR fibers do not preserve formant peaks in rate-based excitation patterns when vowels are presented in background noise (Sachs et al., 1983), 

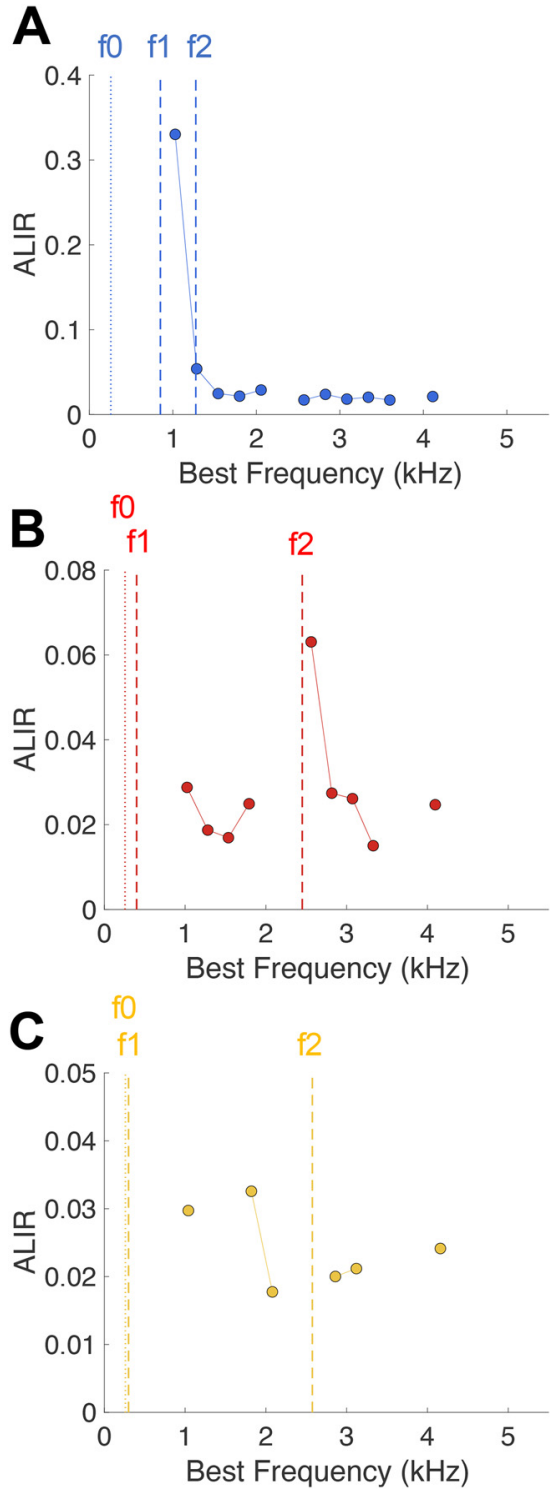

Figure 7. ALIR schemes. A-C, ALIR schemes plotting the average ISIH FFT amplitude around each harmonic of fo for fibers with a BF within $+/-0.5$ octave of the respective fo harmonic, for response to the vowels /a:/ (panel A), /e/ (panel B), and /i/ (panel $\boldsymbol{C}$ ). Formant frequencies are indicated by dashed lines and $f 0$ is indicated as a dotted line for each vowel ALIR scheme.

which was consistent with results presented in the current study. Furthermore, low-SR fibers did not differ from high-SR fibers in terms of rate-based vowel discrimination for naturally-spoken vowels in noise.

In contrast, the temporal responses of low-SR fibers were consistently stronger than those of high-SR fibers in all vowel-evoked responses. This was apparent in the temporal discrimination values (Fig. 3) and in the fluctuation profiles (Fig. 9), with higher $\Delta \mathrm{Cl}$ and more fluctuation for low-SR fibers. This finding agrees with stronger temporal coding in low-SR fibers, as measured by the $\mathrm{Cl}$ to broadband noise stimuli (Louage et al., 2004). Furthermore, low-SR fibers have higher maximum phase-locking values to amplitude modulations than high-SR fibers (Joris and Yin, 1992). Most low-SR fibers in this study had a BF above the maximum phase-locking frequency (i.e., $>4 \mathrm{kHz}$, Versteegh et al., 2011), indicating that they were phase-locking to the lower stimulus frequencies instead of at their BF. It has been hypothesized that low-SR fibers signal mostly to the efferent feedback system, to control cochlear gain, whereas highSR fibers encode the properties of the sound, for further processing toward perception (Carney, 2018). Our data are consistent with this theory, in which high-SR fibers follow perceptual discrimination closely and represent formant frequencies well for the spoken vowels in background noise. However, since low-SR fibers are scarce in the gerbil cochlea, especially among those with a low BF (Ohlemiller and Echteler, 1990; Huet et al., 2016), and are more difficult to hold stable for prolonged recording times than high-SR fibers, the population of low-SR fibers in the current study is perhaps too small to draw conclusions about rate and temporal vowel representation in that population.

\section{Formant frequencies do not all need to be accurately represented in AN fiber spiking patterns to obtain appropriate vowel discrimination}

In the representation schemes that were based on temporal representations, not all formant frequencies of the spoken vowels were represented. Formants seemed to be best represented in the responses to /e/, especially in the ALIR and the dominant component scheme. In the fluctuation profile, the vowel /a:/ showed no peaks at any formant frequency, and in the ALIR of responses to /i/ formant frequencies were not represented. It is interesting that formant representation differs for the different vowels, although they were spoken by the same speaker and presented in the same background noise. In rate-based excitation patterns, formant representation of the vowel /a/ also disappears at lower sound levels compared with those of $/ \mathrm{l} /$ and $/ \varepsilon /$, and $\mathrm{f} 3$ is less well represented for responses to /a/ (Sachs and Young, 1979). One explanation for this observation is that formant representation in the AN fiber population may depend on the amplitude of the formants relative to each other as well as relative to the background noise. As can be seen in Figure 1, the amplitude of $\mathrm{f} 2$ relative to the $\mathrm{f} 1$ amplitude in the vowel spectrum of /e/ is higher than in the vowel spectrum of /i/. Furthermore, the $\mathrm{f} 2$ amplitude of /e/ is well above the background noise whereas $\mathrm{f} 2$ of $/ \mathrm{i} /$ is barely above the background noise (Fig. 1E,F). On the other hand, the frequency and amplitude of $\mathrm{f} 1$ and $\mathrm{f} 2 \mathrm{in}$ the vowel spectrum of /a:/ are much closer together than in /e/ and /i/, and both are well above background noise (Fig. 1D) which means that they should be encoded robustly by phaselocking of many fibers to the same two harmonics. Indeed, in humans, vowel perception is not only dependent on the exact formant frequencies, but also on the relative spectral amplitude of the formants (Kiefte et al., 2010). Hence, AN fiber encoding of formant amplitude and frequency would be required for the correct identification of a specific vowel. However, the specific task investigated here involved discrimination between two vowels, which does not necessitate the correct 
A

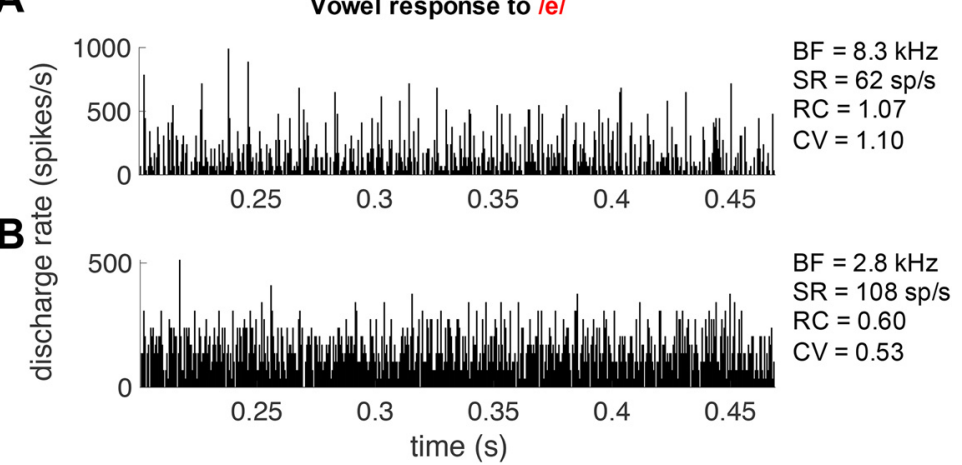

Figure 8. Examples of the PSTH of two fibers during the presentation of the vowel /e/. A, This is an example of a highly fluctuating response. The fiber's BF is far away from the formant frequencies of the vowel /e/ (at $8.3 \mathrm{kHz}$ ). $\boldsymbol{B}$, This is an example of a flat response, showing little fluctuation. The fiber's $B F$ is close to $\mathrm{f} 2(\mathrm{BF}=2.8 \mathrm{kHz}, \mathrm{f} 2=2.5 \mathrm{kHz})$. Both responses derived from high-SR fibers. Fluctuation metrics CV and RC (rate change) are close to twice as high for the fluctuating (panel $\boldsymbol{A}$ ) compared with the flat response (panel $\boldsymbol{B}$ ). The $x$-axis represents the time in seconds relative to the start of the complete stimulus. Only the vowel's analysis window is shown. Bin width is $0.49 \mathrm{~ms}(1 / 8 \times \mathrm{f0})$. Bin height represents the average discharge rate.

identification of both. Indeed, based on the AN data in the current study, this is possible without a completely accurate representation of all formants.

It is an interesting question in itself how the different representation schemes suggested and explored here for the AN population might be integrated and used by the central auditory system. Note, for example, that most representation schemes convert the spike trains of each fiber into one or two dimensions, calculating one or two outcome measures from the (temporal) information in the spike train. It is convenient to reduce dimensionality when comparing between responses from fiber populations,
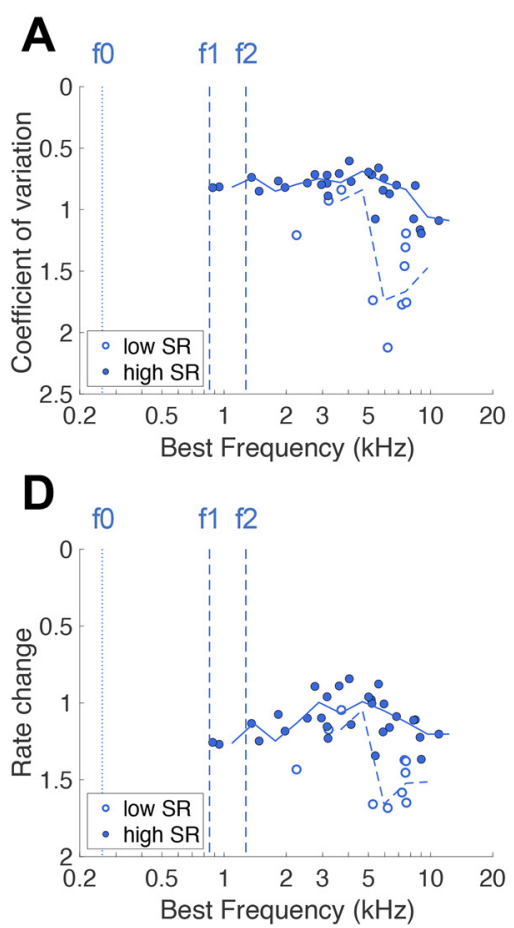
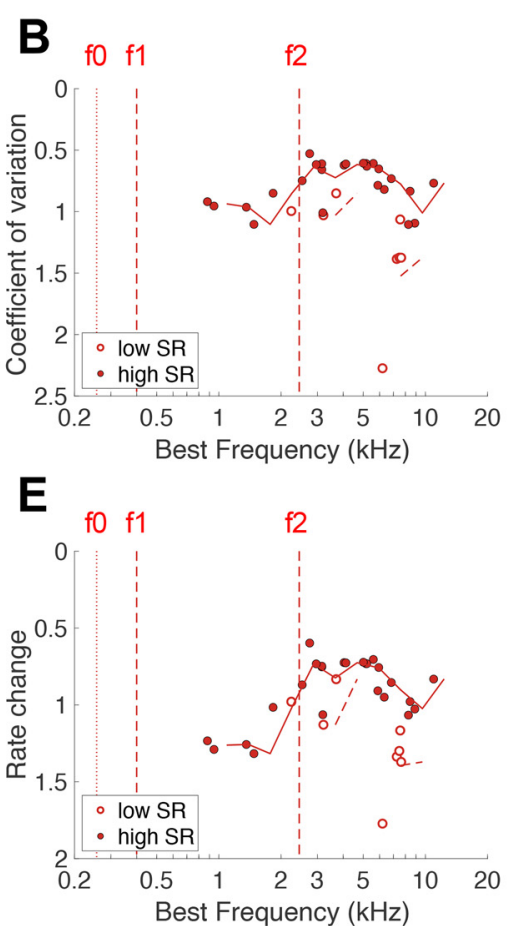
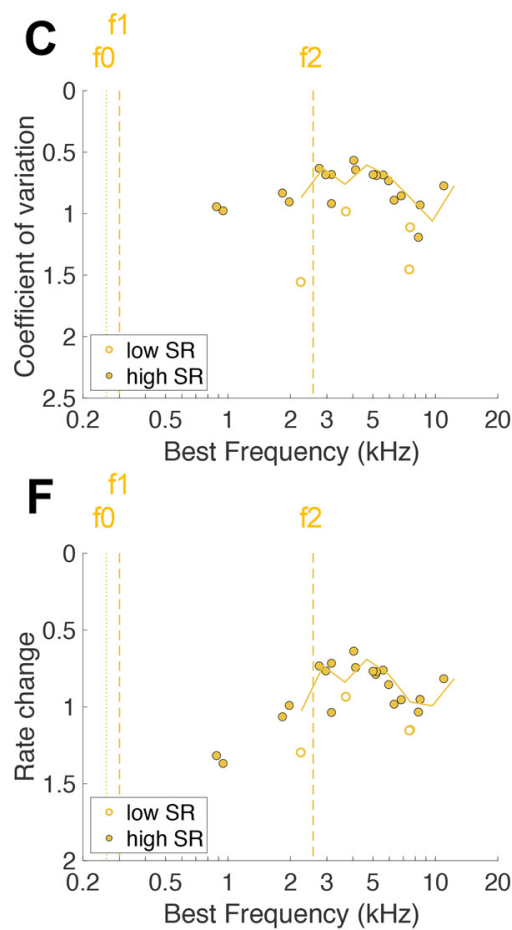

Figure 9. Fluctuation amplitude profiles. $\boldsymbol{A}-\boldsymbol{C}, \mathrm{CV}$ of responses to the vowels /a:/ [panel $\boldsymbol{A}, n=39$ ( $n=10$ low-SR fibers)], /e/ [panel $\boldsymbol{B}, n=33$ ( $n=8$ low-SR fibers)], and /i/ [panel $\boldsymbol{C}, n=24$ ( $n=4$ low-SR fibers)] as a function of the fiber's BF. $\boldsymbol{D}-\boldsymbol{F}$, Rate change, calculated as the mean absolute difference between each bin height divided by the mean bin height of the PSTH, of the three presented vowels. Vowels and unit numbers for panels $\boldsymbol{D}-\boldsymbol{F}$ are the same as in panels $\boldsymbol{A}-\boldsymbol{C}$, respectively. Note that the ordinate is plotted in reverse direction, meaning that lower CVs and rate change values, referring to less fluctuation in the PSTH and thus putatively better formant representation, are higher in the graph. Moving averages of high-SR and low-SR fibers are plotted as solid and dashed lines, respectively. Formant frequencies are indicated by dashed vertical lines and fO as a dotted line for each vowel. 


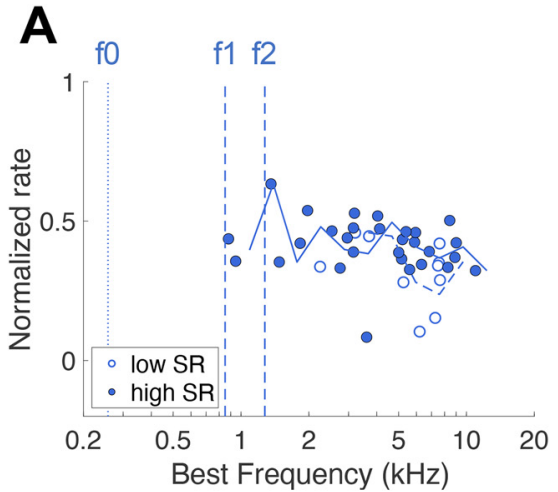

B
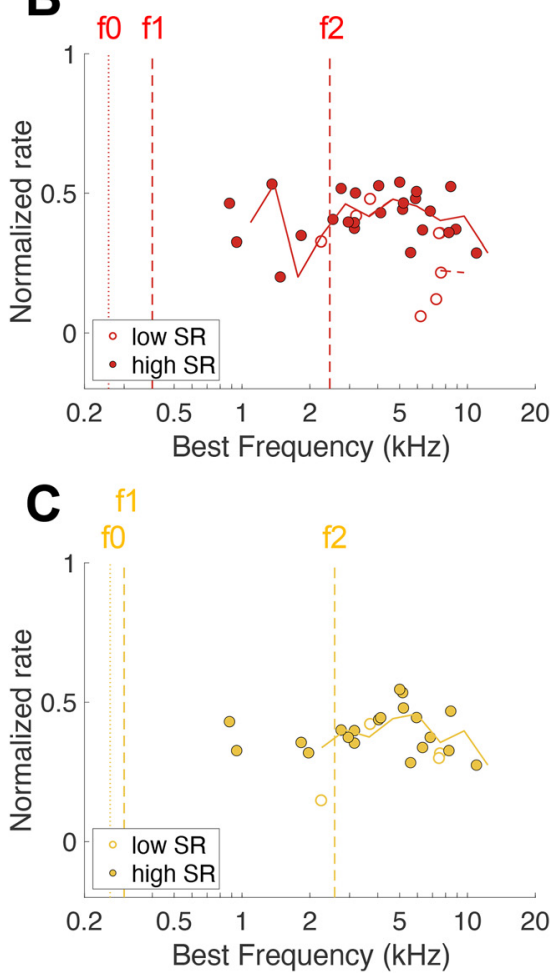

Figure 10. Rate-based excitation patterns. A-C, Rate-based excitation patterns of AN fiber responses to the vowels /a:/ [panel $\boldsymbol{A}, n=39$ ( $n=10$ low-SR fibers)], /e/ [panel $\boldsymbol{B}, n=33(n=8$ low-SR fibers)], and /i/ [panel C, $n=24$ ( $n=4$ low-SR fibers)] plotted as a function of the fiber's BF. Moving averages of highSR and low-SR fibers are plotted as solid and dashed lines, respectively. Formant frequencies are indicated by dashed vertical lines and f0 as a dotted line for each vowel.

vowel responses, or between different conditions, such as with hearing loss. However, each AN fiber branches and projects to many different cell types in the cochlear nucleus. Each cell type extracts different properties from the same spiking pattern, depending on the amount of convergence from different AN fibers, as well as on the physiological and morphologic properties of the cell (Oertel, 1991). It is therefore possible that additional temporal information in the spike trains was available but was not extracted by the applied representation schemes.

In conclusion, this study showed that naturally-spoken vowels in noise were discriminable based on temporal features of AN fiber spike trains but not on the basis of mean discharge rates. This was true both at the singleunit level, contrasting spike trains of the same fiber in response to two different vowels, and at the level of the AN population, looking at representation schemes such as the ALIR, dominant component scheme, and fluctuation profiles. Using perceptual discrimination data of the same species to the same stimuli enabled us to disentangle important aspects of neural vowel encoding in the AN.

\section{References}

Carney LH (2018) Supra-threshold hearing and fluctuation profiles: implications for sensorineural and hidden hearing loss. J Assoc Res Otolaryngol 19:331-352.

Conley RA, Keilson SE (1995) Rate representation and discriminability of second formant frequencies for /epsilon/-like steady-state vowels in cat auditory nerve. J Acoust Soc Am 98:3223-3234.

Delgutte B, Kiang NY (1984a) Speech coding in the auditory nerve: I. Vowel-like sounds. J Acoust Soc Am 75:866-878.

Delgutte B, Kiang NY (1984b) Speech coding in the auditory nerve: V. Vowels in background noise. J Acoust Soc Am 75:908-918.

Diehl RL (2008) Acoustic and auditory phonetics: the adaptive design of speech sound systems. Philos Trans R Soc Lond B Biol Sci 363:965-978.

Dreschler WA, Verschuure H, Ludvigsen C, Westermann S (2001) ICRA noises: artificial noise signals with speech-like spectral and temporal properties for hearing instrument assessment. International Collegium for Rehabilitative Audiology. Audiology 40:148-157.

Green DM, Swets JA (1974) Signal detection theory and psychophysics. New York: Wiley.

Heil P, Neubauer H, Irvine DRF, Brown M (2007) Spontaneous activity of auditory-nerve fibers: insights into stochastic processes at ribbon synapses. J Neurosci 27:8457-8474.

Huet A, Batrel C, Tang Y, Desmadryl G, Wang J, Puel J-L, Bourien J (2016) Sound coding in the auditory nerve of gerbils. Hear Res 338:32-39.

Joris PX (2003) Interaural time sensitivity dominated by cochlea-induced envelope patterns. J Neurosci 23:6345-6350.

Joris PX, Yin TC (1992) Responses to amplitude-modulated tones in the auditory nerve of the cat. J Acoust Soc Am 91:215-232.

Jüchter C, Beutelmann R, Klump GM (2021) Speech sound discrimination by Mongolian gerbils. bioRxiv 471947. doi: 10.1101/2021. 12.10.471947.

Jürgens T, Brand T (2009) Microscopic prediction of speech recognition for listeners with normal hearing in noise using an auditory model. J Acoust Soc Am 126:2635-2648.

Kiefte M, Enright T, Marshall L (2010) The role of formant amplitude in the perception of /i/ and /u/. J Acoust Soc Am 127:2611-2621.

Liberman MC (1978) Auditory-nerve response from cats raised in a low-noise chamber. J Acoust Soc Am 63:442-455.

Liberman MC (1982) Single-neuron labeling in the cat auditory nerve. Science 216:1239-1241.

Louage DHG, van der Heijden M, Joris PX (2004) Temporal properties of responses to broadband noise in the auditory nerve. $J$ Neurophysiol 91:2051-2065.

Merchan-Perez A, Liberman MC (1996) Ultrastructural differences among afferent synapses on cochlear hair cells: correlations with spontaneous discharge rate. J Comp Neurol 371:208-221.

Meyer BT, Jürgens T, Wesker T, Brand T, Kollmeier B (2010) Human phoneme recognition depending on speech-intrinsic variability. $J$ Acoust Soc Am 128:3126-3141.

Oertel D (1991) The role of intrinsic neuronal properties in the encoding of auditory information in the cochlear nuclei. Curr Opin Neurobiol 1:221-228.

Ohlemiller KK, Echteler SM (1990) Functional correlates of characteristic frequency in single cochlear nerve fibers of the Mongolian 
gerbil. J Comp Physiol A Neuroethol Sens Neural Behav Physiol 167:329-338.

Sachs MB, Young ED (1979) Encoding of steady-state vowels in the auditory nerve: representation in terms of discharge rate. J Acoust Soc Am 66:470-479.

Sachs MB, Young ED (1980) Effects of nonlinearities on speech encoding in the auditory nerve. J Acoust Soc Am 68:858-875.

Sachs MB, Voigt HF, Young ED (1983) Auditory nerve representation of vowels in background noise. J Neurophysiol 50:27-45.

Scheidiger C, Carney LH, Dau T, Zaar J (2018) Predicting speech intelligibility based on across-frequency contrast in simulated auditory-nerve fluctuations. Acta Acust United Acust 104:914-917.

Schmiedt RA (1989) Spontaneous rates, thresholds and tuning of auditory-nerve fibers in the gerbil: comparisons to cat data. Hear Res 42:23-35.

Sinex DG, Geisler CD (1983) Responses of auditory-nerve fibers to consonant-vowel syllables. J Acoust Soc Am 73:602-615.
Sinnott JM, Street SL, Mosteller KW, Williamson TL (1997) Behavioral measures of vowel sensitivity in Mongolian gerbils (Meriones unguiculatus): effects of age and genetic origin. Hear Res 112:235-246.

Versteegh CPC, Meenderink SWF, van der Heijden M (2011) Response characteristics in the apex of the gerbil cochlea studied through auditory nerve recordings. J Assoc Res Otolaryngol 12:301-316.

Voigt HF, Sachs MB, Young ED (1982) Representation of whispered vowels in discharge patterns of auditory-nerve fibers. Hear Res 8:49-58.

Winter IM, Robertson D, Yates GK (1990) Diversity of characteristic frequency rate-intensity functions in guinea pig auditory nerve fibres. Hear Res 45:191-202.

Young ED, Sachs MB (1979) Representation of steady-state vowels in the temporal aspects of the discharge patterns of populations of auditory-nerve fibers. J Acoust Soc Am 66:1381-1403. 